\title{
Prognostic value of an automated bone scan index for men with metastatic castration-resistant prostate cancer treated with cabazitaxel
}

Koichi Uemura ${ }^{1,5^{\dagger}}$, Yasuhide Miyoshi ${ }^{1 * \dagger} \mathbb{0}$, Takashi Kawahara ${ }^{1}$, Jikuya Ryosuke ${ }^{1}$, Daisuke Yamashita ${ }^{1}$, Shuko Yoneyama ${ }^{1}$, Yumiko Yokomizo ${ }^{2}$, Kazuki Kobayashi ${ }^{3}$, Takeshi Kishida ${ }^{4}$, Masahiro Yao $^{2}$ and Hiroji Uemura ${ }^{1}$

\begin{abstract}
Background: A computer-assisted diagnostic system for analyzing bone scans (BONENAVI) calculates the automated bone scan index $(\mathrm{aBSI})$. Here we evaluated the aBSI as a prognostic imaging biomarker for men with metastatic castration-resistant prostate cancer (mCRPC) treated with cabazitaxel.

Methods: We retrospectively analyzed 48 patients who received cabazitaxel for mCRPC and evaluated the ability of the aBSI to predict overall survival (OS). The Cox proportional hazards model was used to investigate the associations between baseline aBSI at cabazitaxel treatment and OS with the clinical variables as follows: age, number of cycles of docetaxel, serum prostate-specific antigen, hemoglobin $(\mathrm{Hb})$, lactate dehydrogenase $(\mathrm{LDH})$, and alkaline phosphatase. We determined the C-index to evaluate the discriminatory ability of our models when we included or excluded the aBSI from the analyses.

Results: The median OS after cabazitaxel treatment was 10.0 months, and patients with aBSI $\leq 1 \%$ achieved significantly longer OS compared with patients with $\mathrm{aBSI} \geq 1 \%$. Multivariate analysis showed that age, $\mathrm{Hb}, \mathrm{LDH}$, and aBSI were independent prognostic factors of OS. Adding aBSI to the base model increased the C-index from 0.78 to 0.80 .

Conclusions: The aBSI may serve as a useful imaging biomarker for predicting OS among men with mCRPC treated with cabazitaxel. Prospective studies are required to establish the value of aBSI as prognostic imaging biomarker.
\end{abstract}

Keywords: Prostate cancer, Castration-resistant, Survival prediction, Bone scan index

\section{Background}

Although $80 \%-90 \%$ of prostate cancers with metastasis respond to initial hormone therapy, most patients finally develop to metastatic castration-resistant prostate cancer (mCRPC) $[1,2]$. Sipuleucel-T, abiraterone acetate, enzalutamide, docetaxel, cabazitaxel, and radium-223 have all improved survival among men with $\mathrm{mCRPC}$ and have been approved in western countries [3, 4].

Cabazitaxel is the first chemotherapeutic agent to prolong overall survival (OS) of patients with mCRPC who

\footnotetext{
* Correspondence: miyoyasu@med.yokohama-cu.ac.jp

${ }^{\dagger}$ Equal contributors

${ }^{1}$ Department of Urology and Renal Transplantation, Yokohama City

University Medical Center, 4-57 Urafune-cho, Minami-ku, Yokohama 2320024,

Japan

Full list of author information is available at the end of the article
}

are administered docetaxel. A phase III randomized controlled clinical trial (TROPIC) found that cabazitaxel prolongs OS compared with mitoxantrone and reduces the relative risk of death by $30 \%$ [5]. Accordingly, cabazitaxel is widely used for patients with mCRPC. However, no clinical biomarkers are available for predicting survival.

A computer-assisted diagnostic system for analyzing bone scans (BONENAVI; Fujifilm RI Pharma Co. Ltd., Tokyo, Japan) calculates the automated bone scan index (aBSI) that provides an objective and quantitative evaluation of the burden imposed by bone metastasis [6]. The prognostic value of aBSI as a biomarker was evaluated for predicting the survival of men treated with life-prolonging agents such as enzalutamide [7], abiraterone [7, 8], docetaxel [9-11], and radium-223 [12]. Here we conducted a

(c) The Author(s). 2018 Open Access This article is distributed under the terms of the Creative Commons Attribution 4.0 International License (http://creativecommons.org/licenses/by/4.0/), which permits unrestricted use, distribution, and 
Table 1 Patients' characteristics

\begin{tabular}{ll}
\hline Variables & \\
\hline Median age, years (range) & $71.2(52.5-82.9)$ \\
Cycles of docetaxel, cycles (range) & $9(1-55)$ \\
Use of enzalutamide, $\mathrm{n}(\%)$ & $35(72.9)$ \\
Use of abiraterone acetate, n (\%) & $26(54.1)$ \\
Median aBSI, \%, (range) & $3.5(0.0-12.9)$ \\
Median baseline PSA, ng/mL (range) & $152.1(1.6-3564.0)$ \\
Median baseline Hb, g/dL (range) & $11.0(8.1-14.2)$ \\
Median baseline LDH, IU/L (range) & $262(124-3509)$ \\
Median baseline ALP, IU/L (range) & $414(111-3653)$ \\
Lymph node metastasis, n (\%) & $32(66.6)$ \\
Visceral metastasis, n (\%) & $23(47.9)$ \\
Cycles of cabazitaxel, cycles (range) & $4(1-15)$ \\
Median observation period, months (range) & $7.2(0.6-25.0)$ \\
Cancer death, n (\%) & $25(56.2)$ \\
\hline
\end{tabular}

$a B S I$ automated bone scan index, ALP alkaline phosphatase, $H b$ hemoglobin, $L D H$ lactate dehydrogenase, $P S A$ prostate-specific antigen

retrospective analysis of the relationship between OS and baseline aBSI of patients with mCRPC treated with cabazitaxel.

\section{Methods}

\section{Study design, patients, and treatment}

We retrospectively analyzed 48 patients who were treated with cabazitaxel for bone-metastatic MCRPC between 2014 and 2016 at Yokohama City University
Medical Center, Yokohama City University Hospital, and their associated hospitals. All patients had histologically confirmed prostate adenocarcinoma. The 2009 TNM clinical staging system and the 2005 International Society of Urologic Pathology Gleason grading system were used [13]. The clinical stages of all patients were evaluated using chest and body computed tomography as well as bone scans upon initiation of cabazitaxel treatment.

All patients were initially treated with androgen deprivation therapy (medical or surgical castration with an antiandrogen). After failure to CRPC, all patients were administered enzalutamide, abiraterone, or both, followed by docetaxel with dexamethasone, before cabazitaxel was administered. All patients received continuous androgen ablation therapy (an LH-RH analog) and oral dexamethasone $(0.5-1.0 \mathrm{mg})$. Cabazitaxel treatment was continued until disease progression or unacceptable adverse events occurred.

Some patients received bisphosphonate or denosumab after the development of mCRPC. Patients were not administered sipuleucel- $T$ and radium-223. For terminally ill patients, palliative therapy and pain control using morphine and palliative external beam radiotherapy were used as appropriate. Serum prostatespecific antigen (PSA) levels were measured using the Elecsys Total PSA Assay (Roche Diagnostics Corp., Basle, Switzerland).

\section{Bone scan index}

Bone scan images were obtained within 1 month before or after initiating cabazitaxel treatment and analyzed using

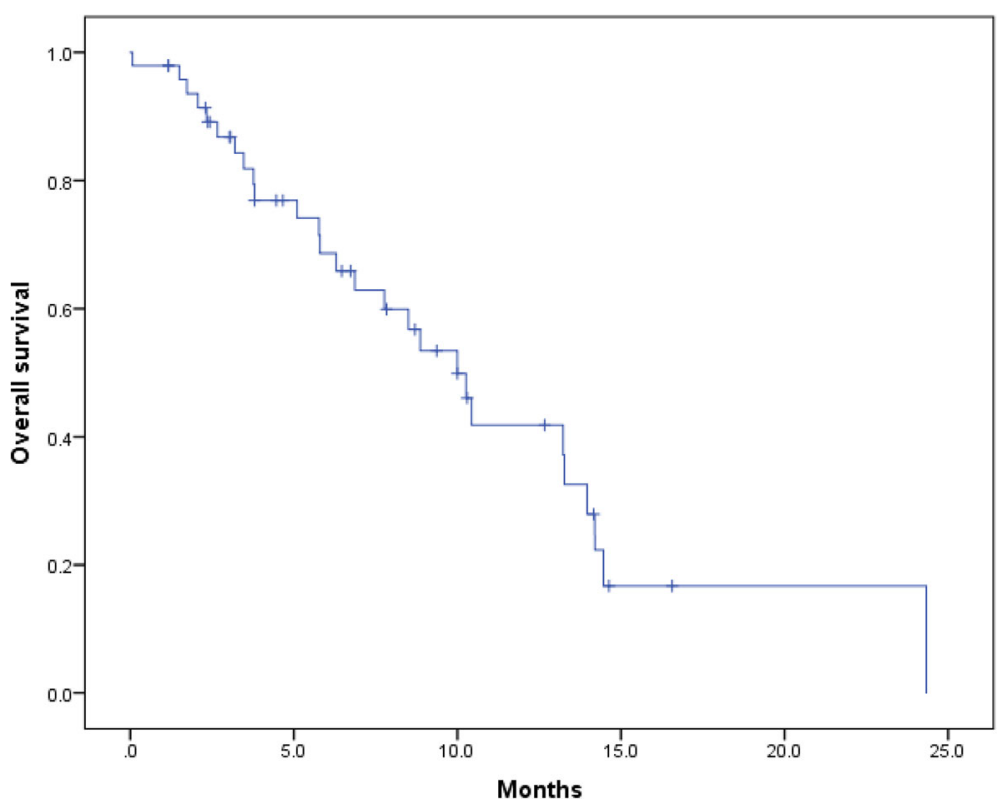

Fig. 1 Kaplan-Meier curve of overall survival (OS) after administration of cabazitaxel. The median OS after cabazitaxel was 10.0 months (95\% confidence interval [Cl], 7.8-12.2 months) 


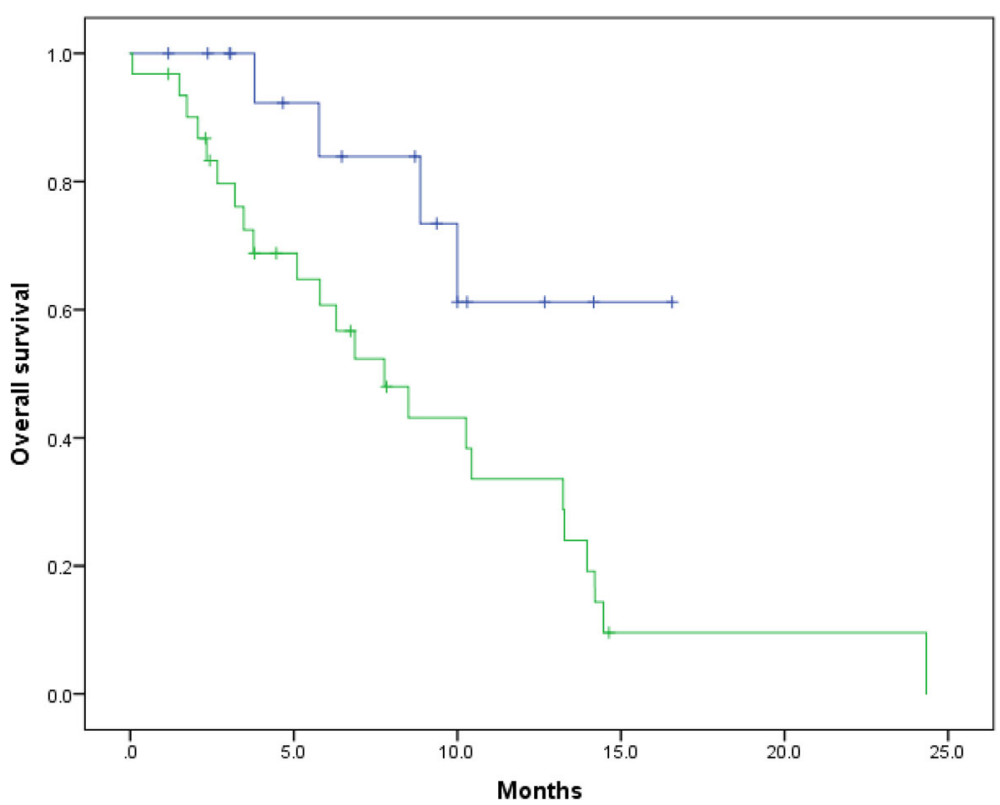

Fig. 2 Kaplan-Meier curves of OS after administration of cabazitaxel according to the automated bone scan index (aBSI). The blue line indicates survival of patients with aBSI $<1 \%(n=17)$, and the green line indicates survival of patients with $\mathrm{aBSI} \geq 1 \%(n=31)$. The median OS of patients with $\mathrm{aBSI}<1 \%$ and $\mathrm{aBSI} \geq 1 \%$ was not reached and 7.8 months $(95 \% \mathrm{Cl}, 4.6-10.9)$, respectively $(p=0.027)$

BONENAVI version 2 [14]. The automated method for analysis of anterior and posterior whole-body bone scan images was previously described [15]. The aBSI was calculated as the percentage of the sum of all hot-spots classified as bone metastases using the artificial neural network (ANN) values [14].

\section{Statistical analysis}

The Cox proportional hazards model with stepwise regression analysis was used to investigate the association between aBSI, OS, and clinical variables of patients treated with cabazitaxel as follows: age, number of cycles of docetaxel, serum PSA, hemoglobin $(\mathrm{Hb})$, lactate dehydrogenase (LDH), and alkaline phosphatase (ALP). The cut-off values for age, number of cycles of docetaxel, serum PSA, $\mathrm{Hb}, \mathrm{LDH}$, and ALP were determined according to the median value of each variable. The cut-off value of aBSI was defined as $1.0 \%$ according to published studies [10, 15]. The relative risks and $95 \%$ confidence intervals (CIs) were calculated, and the C-index was used to assess the discriminatory ability of our models.

The Kaplan-Meier product-limit was used to estimate the survival distribution. The log-rank test was used for the analysis of survival differences. All statistical tests were two-sided, and the significance level was defined as alpha $=0.05$. All analyses were conducted using IBM SPSS Statistics software for Windows, version 24 (IBM Corp., Armonk, NY, USA) and the R package (R Foundation for Statistical Computing, Vienna, Austria).

The experimental procedures were conducted in accordance with the ethical standards of the Helsinki Declaration.

Table 2 Univariate analysis of predictions of overall survival of men with metastatic castration-resistant prostate cancer treated with cabazitaxel

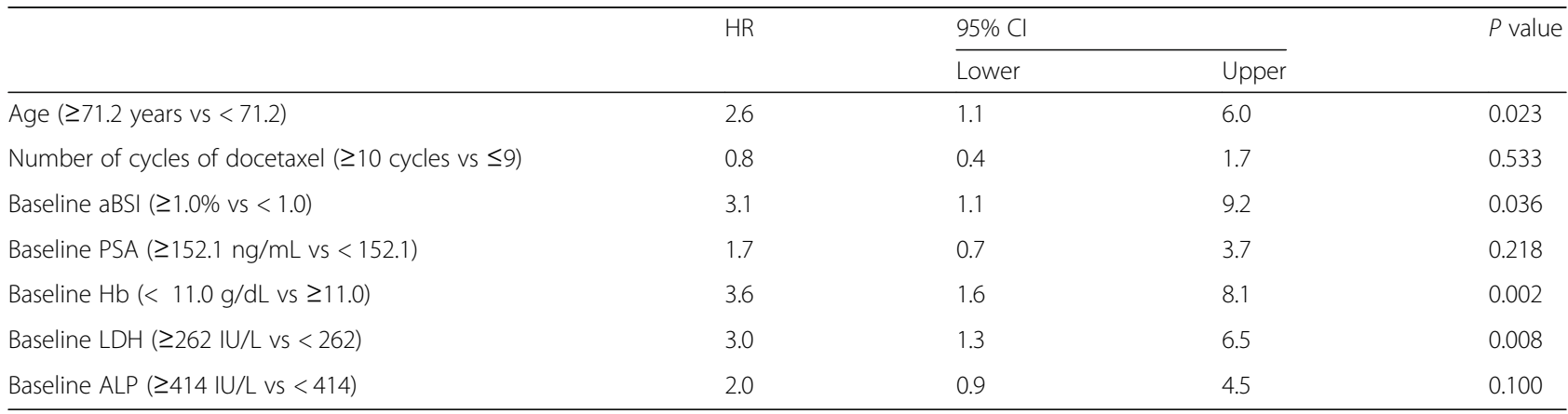

$\overline{a B S I}$ automated bone scan index, $A L P$ alkaline phosphatase, $H b$ hemoglobin, $L D H$ lactate dehydrogenase, $P S A$ prostate-specific antigen 
Table 3 Multivariate analysis of predictions of overall survival of men with metastatic castration-resistant prostate cancer treated with cabazitaxel

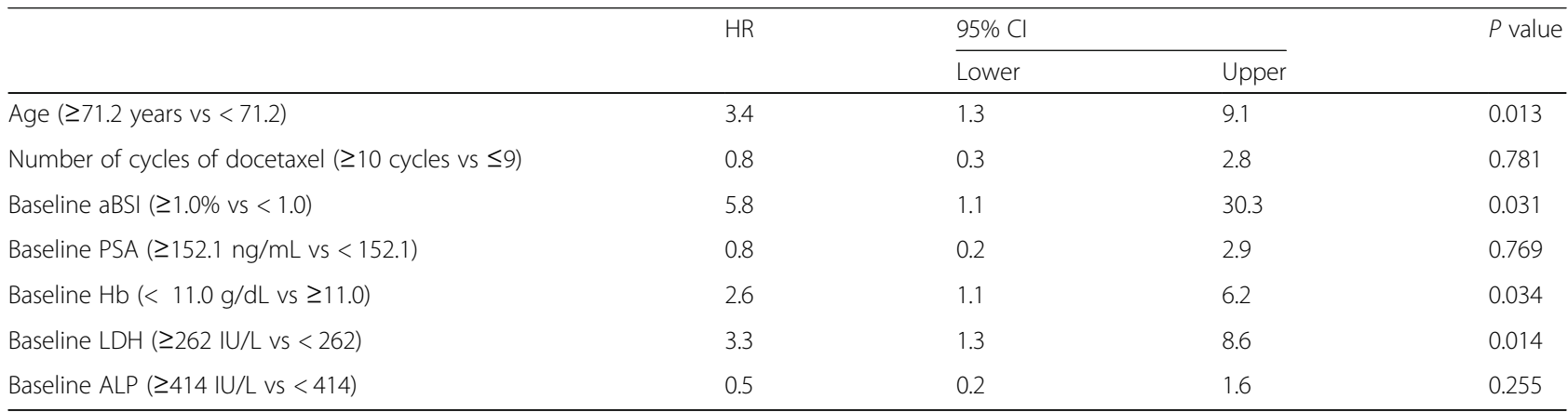

$a B S I$ automated bone scan index, $A L P$ alkaline phosphatase, $H b$ hemoglobin, $L D H$ lactate dehydrogenase, $P S A$ prostate-specific antigen

\section{Results}

Patients' characteristics are shown in Table 1. Among the 48 patients, the sites of metastasis were as follows: 13 , only bone; 12 , lymph node + bone; 3 , visceral + bone; and 20 , visceral + lymph node + bone. The median baseline PSA value was $152.1 \mathrm{ng} / \mathrm{ml}$, and the median aBSI was $3.5 \%$ (range, $0.0 \%-12.9 \%$ ). Twenty-five (56.2\%) patients died, and all deaths were caused by prostate cancer. The median OS after cabazitaxel treatment was initiated was 10.0 months (95\%CI, 7.8-12.2) (Fig. 1). A Kaplan-Meier curve of OS after cabazitaxel treatment as a function of aBSI is shown in Fig. 2. The median OS of patients with aBSI $<1 \%(n=17)$ and $\mathrm{aBSI} \geq 1 \%(n=31)$ was not reached and 7.8 months (95\%CI: 4.6-10.9), respectively. Patients with aBSI $<1 \%$ had a significantly longer OS compared with patients with aBSI $\geq 1 \%$ ( $p=0.027)$.

Univariate analysis revealed that age (hazard ratio [HR], 2.6; 95\%CI, 1.1-6.0; $p=0.023), \mathrm{Hb}$ (HR, 3.6; 95\%CI, 1.6-8.1; $p=0.002)$, LDH (HR, 3.0; 95\%CI 1.3-6.5; $p=0.008)$, and aBSI (HR, 3.1; 95\%CI, $1.1-9.2 ; p=0.036)$ were prognostic factors for OS (Table 2). Multivariate analysis demonstrated that age (HR, 3.4; 95\%CI, 1.3-9.1; $p=0.013)$, $\mathrm{Hb}(\mathrm{HR}, 2.6$; 95\%CI, 1.1-6.2; $p=0.034)$, LDH (HR, 3.3; 95\%CI, 1.3-8.6; $p=0.014$ ), and aBSI (HR, 5.8; $95 \% \mathrm{CI}, 1.1-30.3 ; p=0.031)$ were independent prognostic factors of OS (Table 3 ).

We evaluated the discriminatory ability of our models by determining the $\mathrm{C}$-index. The $\mathrm{C}$-index in our model when aBSI was included was 0.80 for predicting OS after cabazitaxel treatment was initiated. When we analyzed the discriminatory ability of the model after excluding aBSI, we found that the $\mathrm{C}$-index was 0.78 for predicting OS.

Finally, we stratified the patients into cohorts at low risk (0-2 risk factor, $n=26)$ or high risk (3-4 risk factors, $n=22$ ) (Table 4 ). There was a statistically significant difference in OS between these groups $(p<0.001)$ (Fig. 3).

\section{Discussion}

The aBSI is useful imaging biomarker for predicting the survival of men with prostate cancer, including those with hormone-naïve prostate cancer or mCRPC [6, 9, 11, 16-20]. For example, the survival of patients with mCRPC with $\mathrm{aBSI} \geq 3 \%$ is shorter compared with men with aBSI $<3 \%$ who are treated with taxane-based chemotherapy [9]. Further, men with mCRPC with aBSI > $1 \%$ survive for shorter times compared with those with aBSI $\leq 1 \%$ who are treated with docetaxel [10]. Moreover, the aBSI is superior compared with the EOD score as a prognostic imaging biomarker [10].

Recent study demonstrated that not only baseline aBSI before treatment, but also aBSI change after treatment could be useful for prognostic imaging biomarker. Miyoshi et al. reported that decreased aBSI after abirateone acetate or enzalutamide was an independent predictor for longer OS among men with mCRPC [7]. Reza et al. also reported that aBSI change after abirateone acetate was related to survival time in mCRPC patients [8]. On the other hand, Fosbol et al. reported that aBSI was a prognostic biomarker for $\mathrm{mCRPC}$ patients received with Ra-223, although there was no significant association between aBSI change during Ra-223 therapy and OS [12].

Here we analyzed the association between OS, aBSI, and clinical variables of patients with $\mathrm{MCRPC}$ who were treated with cabazitaxel. We identified age, Hb, LDH,

Table 4 Distributions of the risk factors in the low and high risk groups

\begin{tabular}{lllll}
\hline & Age $\geq 71.2$ years & $\mathrm{Hb}<11.0 \mathrm{~g} / \mathrm{dL}$ & $\begin{array}{l}\mathrm{LDH} \geq \\
262 \mathrm{IU} / \mathrm{L}\end{array}$ & $\mathrm{aBSI} \geq 1.0 \%$ \\
\hline $\begin{array}{l}\text { Low risk } \\
(n=26), \mathrm{n}(\%)\end{array}$ & $8(30.8)$ & $5(19.2)$ & $6(23.1)$ & $13(50.0)$ \\
$\begin{array}{l}\text { High risk } \\
(n=22), \mathrm{n}(\%)\end{array}$ & $16(72.7)$ & $18(81.8)$ & $18(81.8)$ & $18(81.8)$ \\
\hline
\end{tabular}

$\overline{a B S}$ automated bone scan index, $\mathrm{Hb}$ hemoglobin, $\mathrm{LDH}$ lactate dehydrogenase 


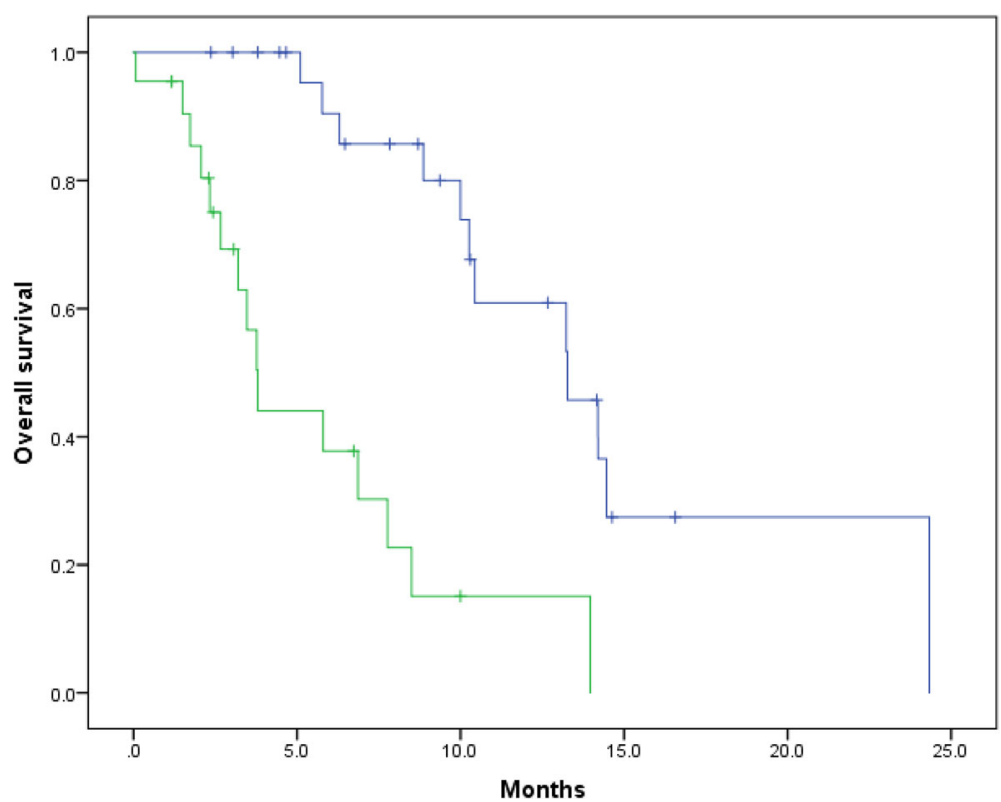

Fig. 3 Kaplan-Meier curves of OS after initiating cabazitaxel treatment according to risk group. We stratified the patients into cohorts at low risk $(0-2$ risk factor, $n=26)$ and high risk (3-4 risk factors, $n=22)$. The blue and green lines indicate survival of patients at low and high risk, respectively. The median OS values of patients at low and high risk were 13.3 months and 3.8 months, respectively $(p<0.001)$. The incidences of the risk factors of each group are shown in Table 4

and aBSI as independent prognostic factors of OS. Including the aBSI in the base model improved the $\mathrm{C}$ index from 0.78 to 0.80 . Therefore, the aBSI may serve as a promising imaging biomarker for predicting OS among men with $\mathrm{MCRPC}$ who are treated with cabazitaxel. Further, the aBSI may provide useful information about such patients.

Although we show here that the aBSI played an important role in evaluating bone metastases, our study has several limitations. For example, our study was retrospective, the number of subjects was small, and the observation periods were relatively short. Prospective evaluation of larger patient populations and longer observations are warranted to establish the usefulness of the aBSI as a prognostic imaging biomarker.

\section{Conclusion}

Our analyses strongly indicate that the aBSI may serve as a useful tool for risk stratification of patients with $\mathrm{mCRPC}$ undergoing treatment with cabazitaxel.

\section{Abbreviations}

aBSI: Automated bone scan index; ALP: Alkaline phosphatase; $\mathrm{Cl}$ : Confidence interval; EOD: Extent of disease; Hb: Hemoglobin; HR: Hazard ratio; LDH: Lactate dehydrogenase; mCRPC: Metastatic castration-resistant prostate cancer; OS: Overall survival; PSA: Prostate-specific antigen

\section{Availability of data and materials}

All data used for this study are available from the corresponding author upon reasonable request.

\section{Authors' contributions}

YM designed the study. YM and KU contributed to statistical analysis and wrote the manuscript. TK, JR, DY, SY, YY, KK, TK, HU, and MY supervised the study. All authors have read and approved the final manuscript.

\section{Ethics approval and consent to participate}

The Institutional Review Board of Yokohama City University Medical Center approved this study. Informed consent to participate in the study was obtained from the subjects.

\section{Competing interests}

The authors declare that they have no competing interests.

\section{Publisher's Note}

Springer Nature remains neutral with regard to jurisdictional claims in published maps and institutional affiliations.

\section{Author details}

'Department of Urology and Renal Transplantation, Yokohama City University Medical Center, 4-57 Urafune-cho, Minami-ku, Yokohama 2320024, Japan. ${ }^{2}$ Department of Urology, Yokohama City University Graduate School of Medicine, Yokohama, Japan. ${ }^{3}$ Department of Urology, Yokosuka Kyosai Hospital, Yokosuka, Japan. ${ }^{4}$ Department of Urology, Kanagawa Cancer Center, Yokohama, Japan. ${ }^{5}$ Department of Urology, Yokohama City Minato Red Cross Hospital, Yokohama, Japan.

Received: 28 December 2017 Accepted: 18 April 2018 Published online: 02 May 2018

\section{References}

1. Small EJ, Vogelzang NJ. Second-line hormonal therapy for advanced prostate cancer: a shifting paradigm. J Clin Oncol. 1997;15(1):382-8.

2. Oh WK, Kantoff PW. Management of hormone refractory prostate cancer: current standards and future prospects. J Urol. 1998;160(4):1220-9.

3. Agarwal N, Di Lorenzo G, Sonpavde G, Bellmunt J. New agents for prostate cancer. Ann Oncol. 2014;25(9):1700-9. 
4. Sartor O, de Bono JS. Metastatic prostate Cancer. N Engl J Med. 2018;378(7): 645-57.

5. de Bono JS, Oudard S, Ozguroglu M, Hansen S, Machiels JP, Kocak I, Gravis G, Bodrogi I, Mackenzie MJ, Shen L, et al. Prednisone plus cabazitaxel or mitoxantrone for metastatic castration-resistant prostate cancer progressing after docetaxel treatment: a randomised open-label trial. Lancet (London, England). 2010;376(9747):1147-54.

6. Horikoshi H, Kikuchi A, Onoguchi M, Sjostrand K, Edenbrandt L. Computeraided diagnosis system for bone scintigrams from Japanese patients: importance of training database. Ann Nucl Med. 2012;26(8):622-6.

7. Miyoshi Y, Uemura K, Kawahara T, Yoneyama S, Hattori Y, Teranishi Jl, Ohta $\mathrm{Jl}$, Takebayashi S, Yokomizo Y, Hayashi N, et al. Prognostic value of automated bone scan index in men with metastatic castration-resistant prostate Cancer treated with Enzalutamide or Abiraterone acetate. Clin Genitourin Cancer. 2017;15(4):472-8.

8. Reza M, Ohlsson M, Kaboteh R, Anand A, Franck-Lissbrant I, Damber JE, Widmark A, Thellenberg-Karlsson C, Budaus L, Steuber T, et al. Bone scan index as an imaging biomarker in metastatic castration-resistant prostate Cancer: a multicentre study based on patients treated with Abiraterone acetate (Zytiga) in clinical practice. Eur Urol Focus. 2016;2(5):540-6.

9. Mitsui $Y$, Shiina H, Yamamoto Y, Haramoto M, Arichi N, Yasumoto H, Kitagaki $\mathrm{H}$, Igawa M. Prediction of survival benefit using an automated bone scan index in patients with castration-resistant prostate cancer. BJU Int. 2012; 110(11 Pt B):E628-34.

10. Uemura K, Miyoshi Y, Kawahara T, Yoneyama S, Hattori Y, Teranishi J, Kondo K, Moriyama M, Takebayashi S, Yokomizo Y, et al. Prognostic value of a computer-aided diagnosis system involving bone scans among men treated with docetaxel for metastatic castration-resistant prostate cancer. BMC Cancer. 2016;16:109.

11. Kaboteh R, Gjertsson P, Leek H, Lomsky M, Ohlsson M, Sjostrand K, Edenbrandt L. Progression of bone metastases in patients with prostate cancer - automated detection of new lesions and calculation of bone scan index. EJNMMI Res. 2013;3(1):64.

12. Fosbol MO, Petersen PM, Daugaard G, Holm S, Kjaer A, Mortensen J. Impact of treatment delay in Radium-223 therapy of metastatic castration-resistant prostate cancer patients. Ann Nucl Med. 2018:32(1):16-21.

13. Epstein Jl, Allsbrook WC Jr, Amin MB, Egevad LL, Committee IG. The 2005 International Society of Urological Pathology (ISUP) consensus conference on Gleason grading of prostatic carcinoma. Am J Surg Pathol. 2005;29(9):1228-42.

14. Koizumi M, Wagatsuma K, Miyaji N, Murata T, Miwa K, Takiguchi T, Makino T, Koyama M. Evaluation of a computer-assisted diagnosis system, BONENAVI version 2, for bone scintigraphy in cancer patients in a routine clinical setting. Ann Nucl Med. 2015;29(2):138-48.

15. Ulmert D, Kaboteh R, Fox JJ, Savage C, Evans MJ, Lilja H, Abrahamsson PA, Bjork T, Gerdtsson A, Bjartell A, et al. A novel automated platform for quantifying the extent of skeletal tumour involvement in prostate cancer patients using the bone scan index. Eur Urol. 2012;62(1):78-84.

16. Armstrong AJ, Kaboteh R, Carducci MA, Damber JE, Stadler WM, Hansen M, Edenbrandt L, Forsberg G, Nordle O, Pili R, et al. Assessment of the bone scan index in a randomized placebo-controlled trial of tasquinimod in men with metastatic castration-resistant prostate cancer (mCRPC). Urol Oncol. 2014;32(8):1308-16.

17. Dennis ER, Jia X, Mezheritskiy IS, Stephenson RD, Schoder H, Fox JJ, Heller G, Scher HI, Larson SM, Morris MJ. Bone scan index: a quantitative treatment response biomarker for castration-resistant metastatic prostate cancer. J Clin Oncol. 2012;30(5):519-24.

18. Kaboteh R, Damber JE, Gjertsson P, Hoglund P, Lomsky M, Ohlsson M, Edenbrandt L. Bone scan index: a prognostic imaging biomarker for highrisk prostate cancer patients receiving primary hormonal therapy. EJNMMI Res. 2013;3(1):9.

19. Kalderstam J, Sadik M, Edenbrandt L, Ohlsson M. Analysis of regional bone scan index measurements for the survival of patients with prostate cancer BMC Med Imaging. 2014;14:24.

20. Poulsen MH, Rasmussen J, Edenbrandt L, Hoilund-Carlsen PF, Gerke O, Johansen A, Lund L. Bone scan index predicts outcome in patients with metastatic hormone sensitive prostate cancer. BJU Int. 2015;

\section{Ready to submit your research? Choose BMC and benefit from:}

- fast, convenient online submission

- thorough peer review by experienced researchers in your field

- rapid publication on acceptance

- support for research data, including large and complex data types

- gold Open Access which fosters wider collaboration and increased citations

- maximum visibility for your research: over $100 \mathrm{M}$ website views per year

At BMC, research is always in progress.

Learn more biomedcentral.com/submissions 